\title{
Graphene-Conducting Polymer Nanocomposites for Enhancing Electrochemical Capacitive Energy Storage
}

Shen, Fei; Pankratov, Dmitry; Chi, Qijin

Published in:

Current Opinion in Electrochemistry

Link to article, DOI:

10.1016/j.coelec.2017.10.023

Publication date:

2017

Document Version

Peer reviewed version

Link back to DTU Orbit

Citation (APA):

Shen, F., Pankratov, D., \& Chi, Q. (2017). Graphene-Conducting Polymer Nanocomposites for Enhancing Electrochemical Capacitive Energy Storage. Current Opinion in Electrochemistry, 4(1), 133-144.

https://doi.org/10.1016/j.coelec.2017.10.023

\section{General rights}

Copyright and moral rights for the publications made accessible in the public portal are retained by the authors and/or other copyright owners and it is a condition of accessing publications that users recognise and abide by the legal requirements associated with these rights.

- Users may download and print one copy of any publication from the public portal for the purpose of private study or research.

- You may not further distribute the material or use it for any profit-making activity or commercial gain

- You may freely distribute the URL identifying the publication in the public portal 
[Type the document title]

October 8, 2017

\section{Highlights}

- Recent developments of graphene-conducting polymer nanocomposites are overviewed.

- Material design, synthesis and applications in flexible supercapacitors are highlighted.

- Current challenges and future perspective are briefly commented. 


\title{
Graphene-Conducting Polymer Nanocomposites for Enhancing Electrochemical Capacitive Energy Storage
}

\author{
Fei Shen ${ }^{\dagger}$, Dmitry Pankratov ${ }^{\dagger}$, and Qijin Chi*
}

Department of Chemistry, Technical University of Denmark, DK-2800 Kongens Lyngby, Denmark. *Corresponding author. E-mail: cq@ kemi.dtu.dk

† These authors contributed equally to this work.

Abstract: The evolution of power generation, expansion of transportation and electrification, and popularization of portable electronic devices have altogether posed growing demands for more efficient energy storage systems. Supercapacitors, as one of major electrochemical energy storage devices, have recently received intensive attention. In this minireview, our focus is on graphene-conducting polymer nanocomposites and their applications in supercapacitors that have potential to perform high power and energy density, fast charge/discharge rate, low cost and ecofriendly operation conditions. We first introduce major types of supercapacitors and their working principles. We then overview several hybrid material systems combing graphene and various conducting polymers such as polyaniline, polythiophene, polypyrrole and their derivatives, with the emphasis on the composite design, synthesis methods, capacitive performance and current applications in flexible and bendable supercapacitors. Finally, the challenges and perspective of such composite electrode materials for supercapacitors are commented for perspective of further research directions.

Keywords: Supercapacitor, electrochemical energy storage, graphene, conducting polymer, nanostructured composite materials 
[Type the document title]

October 8, 2017

\section{Introduction}

An immense demand towards portable electronic devices and hybrid electric vehicles has raised unprecedented requirements to current energy storage systems (ESSs). As the most common energy storage devices, batteries have been the technology of choice for supplying power for most applications in the past long time, because they can store large amount of energy in a relatively small volume and less weight [1]. However, the low power density and short cycle life of batteries can hardly meet the demand of new-energy sectors. Supercapacitors (SCs), with high power density, fast charge/discharge rate, long-cycle life and environmentally friendly operation, hold great potential to compensate the shortcomings of batteries and offer additional flexibility for storage of electricity) [2]. Moreover, the combination of SCs (high power density) and batteries (high energy density) could further provide opportunity to build more advanced hybrid ESSs.

SCs, also called electrochemical capacitors (ECs), are rechargeable electrochemical energy storage devices capable of providing up to thousand times higher power output compared to the batteries of similar size [3]. Although SCs store lower amount of energy than batteries, in most cases, their energy density is still much higher than that of conventional capacitors [4]. In addition, SCs can be fully charged or discharged in seconds, while batteries take much longer time due to electrochemical kinetics through a polarization resistance [5]. The performance of various energy storage devices is compared in the Ragone plot (Figure 1). As seen from the plot, SCs could fill the gap between conventional capacitors and batteries. Therefore, SCs could play a crucial role in the development of new ESSs. 
[Type the document title]

October 8, 2017



Figure 1. Ragone plot comparing specific energy and power capability of conventional capacitors, electrochemical capacitors (supercapacitors) and various batteries. Times shown are the time constants of the devices, obtained by dividing the energy density by the power. Adapted from ref. [6]

\section{Working principles and classification}

In a conventional capacitor, two electrically conductive electrodes are separated by an insulating dielectric material (Figure 2a), and the capacitance is originated in pure electrostatic charges. In contrast, SCs are composed of two electrodes mechanically separated by an ion permeable insulating membrane, but ionically connected to an electrolyte (Figure 2b). Each electrode/electrolyte interface represents a capacitor, a SC can thus be regarded as two capacitors in series. If two capacitors exhibit the same behavior, i.e. using the same material for both electrodes, then such SC is termed as a symmetric SC. Alternatively, two different electrodes can construct an asymmetric capacitor. The overall specific capacitance $\left(\mathrm{C}_{S}\right)$ of a $\mathrm{SC}$ can be calculated by $\mathrm{Eq}(1)$. 
[Type the document title]

\section{October 8, 2017}

$$
C_{S}=\frac{C_{1} * C_{2}}{C_{1}+C_{2}}
$$

where $C_{1}$ and $C_{2}$ are the specific capacitance of two electrodes, respectively. In addition, the energy $(E)$ density that a SC enables to store can be expressed by $\mathrm{Eq}(2)$.

$$
E=\frac{1}{2} C V^{2}
$$

where $V$ is the applied cell voltage. Equation (2) shows that the specific capacitance and the applicable cell voltage determine the output energy of a SC. In order to enhance the energy density of a SC, we need to design electrode materials that enable to increase the device capacitance or expand the applicable voltage window. The maximum power $\left(P_{\max }\right)$ of a SC can be calculated according to $\mathrm{Eq}(3)$.

$$
P_{\max }=\frac{V^{2}}{4 R}
$$

where $R$ is the equivalent series resistance. Hence, the power output is determined by the cell voltage and internal resistance.



Figure 2. Schematic diagrams of (a) a conventional capacitor and (b) structure and working principles of a symmetric supercapacitor 
[Type the document title]

October 8, 2017

To sum up, the capacitance mainly depends on the electrode materials, and the available cell voltage window depends on both of electrode material and electrolyte. Therefore, the electrode materials and electrolytes are two critical factors to the specific energy and power of SCs. Some electrode materials are discussed in detail in the following sections. As for the electrolytes used in SCs, briefly, aqueous electrolytes have high ionic conductivity (up to $\sim 1 \mathrm{~S}$ $\left.\mathrm{cm}^{-1}\right)$ but relatively low decomposition voltage $(\sim 1.23 \mathrm{~V})$; while organic electrolytes allow the use of cell operating voltage up to $\sim 3-4 \mathrm{~V}$ but they could induce higher electrical resistivity, resulting in a higher internal resistance [7].

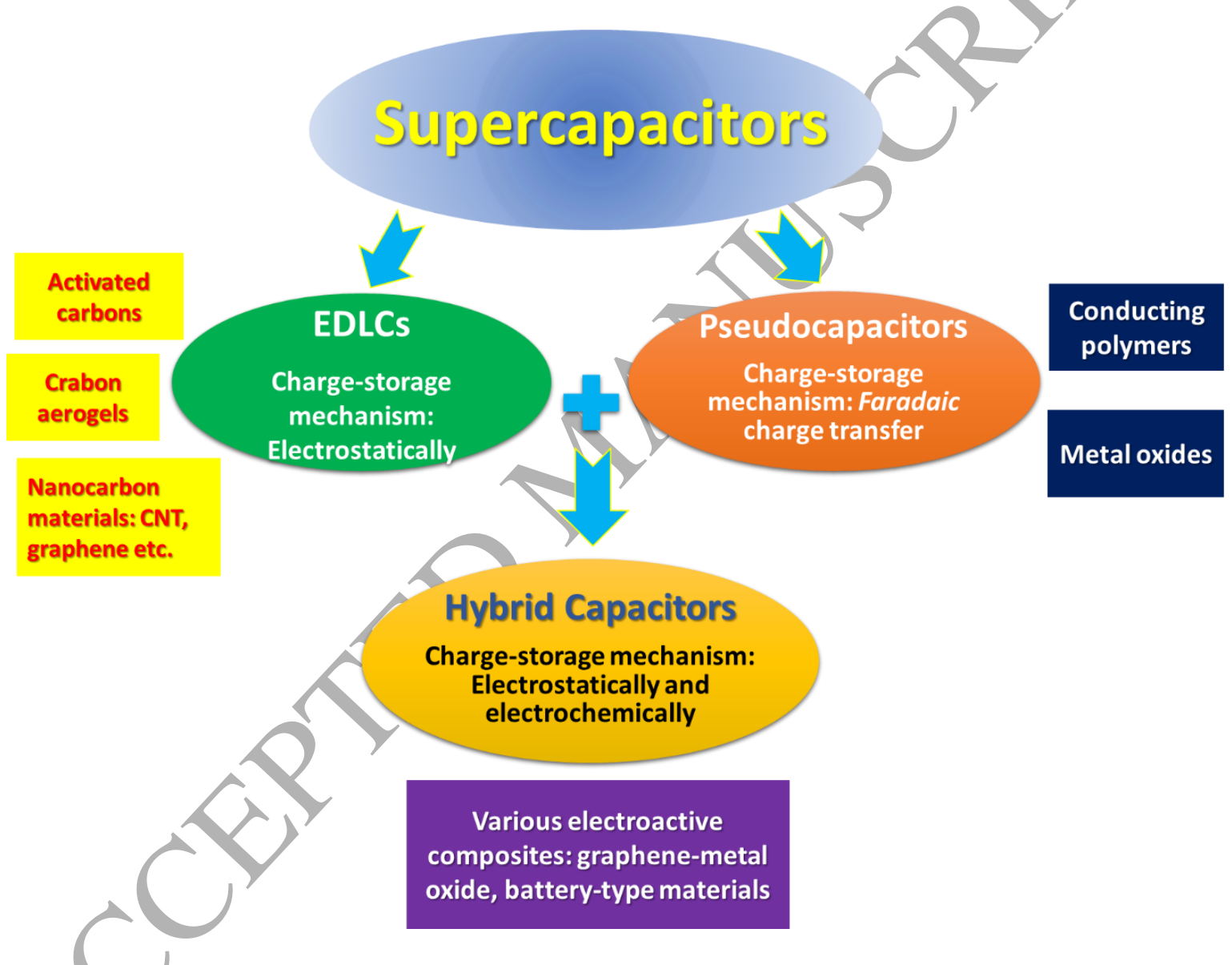

Figure 3. Diagram representations for three types of supercapacitors and corresponding most common materials used in the construction.

The working principles of SCs also depend on the type of electrode materials, which is electroactive or not. The charge storage of SCs arises from electric double layer (ELD) induced by ion adsorption or/and faradic redox processes of electroactive species at the electrode/electrolyte interface (Figure 2b). According to the charge-storage mechanism, SCs 
[Type the document title]

October 8, 2017

can be generally classified into three types, i.e. electric double layer capacitors (EDLCs), pseudocapacitors and hybrid capacitors (Figure 3). In the first-type SCs, reversible electrostatic attraction occurs between the ions from electrolytes and the charged surface of electrodes. This kind of supercapacitors can be called as EDLCs, which have much higher capacitance values than conventional capacitors due to the large surface area of electrodes (up to thousands of $\mathrm{m}^{2} \mathrm{~g}^{-1}$ ) and the extremely thin double-layer distance (e.g. a few tenths of nanometer) [8]. The double layer capacitance of an EDLC can be expressed as:

$$
C=\frac{\varepsilon_{r} \varepsilon_{0} A}{d}
$$

where $\varepsilon_{\mathrm{r}}$ and $\varepsilon_{0}$ are the dielectric constant of electrolyte and free space (or vacuum), respectively; $A$ is the specific surface area of electrode, and $d$ is the thickness of electric double layer. Carbonaceous materials have so far been the most widely used electrode substrate, due to their high specific surface area, high chemical stability and relatively low cost (Figure 3). During the charging process, cations are driven to accumulate on the negative electrode and anions on the positive electrode forming a double layer for energy storage. During the discharging process, in contrast, ions travel away from the surface of electrode and mixed in electrolyte. There is no charge transfer in the process, which guarantees very fast charge/discharge rate and better power performance of EDLCs. However, this kind of SCs normally suffer from low energy density.

In pseudocapacitors, fast and reversible faradic reactions take place at the interface during the energy storage and delivery process accompanied by double layer charge storage; hence, they generally exhibit much higher specific capacitance and energy density than EDLCs. For a pseudocapacitor, its capacitance can be described as:

$$
C=\frac{\Delta Q}{\Delta V}
$$

where $Q$ is the accumulated charge and $V$ is the applied voltage. As the redox processes in a pseudocapacitor occur mainly at the electrode surface, the charge/discharge rates are faster than that of a battery where redox reactions occur within the electrode itself [9]. Conducting polymers and metal oxides/sulfides are among the most common electroactive materials used in constructing pseudocapacitors (Figure 3). Compared to metal oxides/sulfides, conducting polymers have shown some unique physicochemical properties, such as larger theoretical specific capacitance, better electrical conductivity, lower cost and ease of large-scale 
[Type the document title]

October 8, 2017

production [10]. In conducting polymers based pseudocapacitors, the charge/discharge processes are associated with the doping/de-doping processes of conducting polymers. There are two types of doping organic polymers, i.e. p-doping (oxidation) and n-doping (reduction), which could enhance the overall conductivity of a polymer up to the metallic level [11]. The p-doping of polymers normally refers to the partial oxidation of the polymers $(\mathrm{P})$, and counteranions $\left(\mathrm{A}^{-}\right)$are intercalated to preserve electrical neutrality in the system, $\mathrm{Eq}(6)$. In contrast, the n-doping of polymers involves the partial reduction of the polymer and insertion of counter-cations $\left(\mathrm{M}^{+}\right)$to preserve electrical neutrality, $\mathrm{Eq}(7)$.

$$
\begin{aligned}
& \mathrm{P}_{m}-x e^{-}+x \mathrm{~A}^{-} \stackrel{\text { Charge/Discharge }}{\longleftrightarrow} \mathrm{P}_{m}^{x+} \mathrm{A}_{x}^{-} \\
& \mathrm{P}_{m}+x e^{-}+x \mathrm{M}^{+} \stackrel{\text { Charge/Discharge }}{\longleftrightarrow} \mathrm{P}_{m}^{x-} \mathrm{M}_{x}^{+}
\end{aligned}
$$

where $\mathrm{m}$ is the polymerization degree and $\mathrm{x}$ is the number of charges transferred. However, only using these redox materials in capacitors encounters other problems such as poor stability and low power density.

In order to overcome the drawbacks of EDCLs and pseudocapacitors, both electrode materials (carbonaceous materials and redox-active materials) can be used in construction of a SC. Such SCs is normally termed a hybrid SC (Figure 3). In general, carbon materials are used as the scaffold to support electroactive components, and their composites act as electrodes of a hybrid SC. Hybrid SCs could display enhanced energy density, high power density and relatively high stability. In the following sections, we will focus on highlighting recent advances in the development of graphene-conducting polymer nanocomposite based SCs and their potential applications in flexible electronics.

\section{Advantages of graphene-conducting polymer nanocomposites as supercapacitor electrode materials}

Graphene (Gr), a two-dimensional monolayer of $\mathrm{sp}^{2}$-bonded carbon atoms, exhibits a range of remarkable physical properties, including high elastic modulus and fracture strength $(\sim 1 \mathrm{TPa}$ and $\sim 130 \mathrm{GPa}$, respectively), good electrical conductivity $\left(\sim 10^{4}-10^{5} \mathrm{~S} \mathrm{~m}^{-1}\right)$, and very high surface area $\left(\sim 2600 \mathrm{~m}^{2} \mathrm{~g}^{-1}\right)$ [12]. Double layer capacitance of pristine single-layered graphene was reported to be about $21 \mu \mathrm{F} \mathrm{cm}^{-2}$ [13], therefore the maximum specific capacitance of $\sim 550$ 
[Type the document title]

October 8, 2017

$\mathrm{F} \mathrm{g}^{-1}$ can be expected if the entire specific surface area of graphene nanosheets are effectively used. A specific capacitance of $513 \mathrm{~F} \mathrm{~g}^{-1}$ has recently been achieved for the three-dimensional (3D) reduced graphene oxide ( $\mathrm{rGO}$ ) networks fabricated on a copper foam surface [14]. Doping of Gr nanosheets with heteroatoms, e.g. N, B, or S, can modify the charge distribution and hence further enhance the specific capacitance and power density of graphene supported SCs [15-17].

Conducting conjugated polymers (CPs), e.g. polyaniline (PAni), polythiophene (PT), polypyrrole (PPy) and their derivatives, are prospective materials for charge- and energystorage applications due to their relatively low cost, good electrical conductivity (up to $4.6 \cdot 10^{5} \mathrm{~S} \mathrm{~m}^{-1}[18]$ ) and high capacitance, mainly related to the pseudo-faradaic phenomena (up to $2000 \mathrm{~F} \mathrm{~g}^{-1}$ for PAni [19], $485 \mathrm{~F} \mathrm{~g}^{-1}$ for PT [20], $620 \mathrm{~F} \mathrm{~g}^{-1}$ for PPy [21], dependent on the doping level). Although CPs hold promising characteristics for electrochemical energy storage, however, their low stability under charge/discharge conditions has limited practical applications [20]. The rapid decayed stability is mainly caused by considerable mechanical degradation (e.g. swelling and shrinking) and irreversible structural changes occurred during the charge-discharge processes.

Recent studies have shown that Gr can affect the molecular conformation and orientation of $\mathrm{CP}$ chains, leading to the positive synergistic effect on their composites to achieve higher electrical conductivity and higher stability than individual components [22]. Other advantages of Gr-CP composite materials include high surface area (for enhancement of the specific capacitance), considerable structural diversity, small size of particles and short distance for ion transfer, structural uniformity and controllable morphology [23, 24]. Moreover, the integration of CP with $\mathrm{Gr}$ prevents self-aggregation or re-stacking of graphene nanosheets to avoid decrease in the specific surface area and irreversible capacitance loss arising from changes in the nanocomposite morphology.

\subsection{Material Design, Synthesis methods, and Performance}

Several crucial aspects have to be considered in the development of Gr/CP nanocomposites, including $i$ ) appropriate methods of synthesis, ii) surface compatibility of $\mathrm{CP}$ with Gr, iii) controlling thickness and morphology of the $\mathrm{CP}$ film, and $i v$ ) surface properties of the nanocomposite material (e.g. surface roughness, hydrophilicity) [25]. 
[Type the document title]

October 8, 2017

There are two major strategic approaches for preparation of $\mathrm{Gr} / \mathrm{CP}$ nanocomposites, viz. through incorporation of the $\mathrm{CP}$ between the $\mathrm{Gr}$ sheets (2D nanocomposites) and employment of $\mathrm{Gr}$ nanosheets as a matrix for the synthesis of $\mathrm{CP}$ or $\mathrm{Gr} / \mathrm{CP}$ nanostructures with various morphology (3D networked materials) [26]. This can be achieved by direct incorporation of $\mathrm{CP}$ nanostructures during the GO reduction, in situ polymerization of monomers on rGO surface or template synthesis, in which metal or polymer with micro-/nano structures are used for layer-by-layer assembly of Gr and CP followed by sequent removal of templates [27]. A variety of methods can be used for the synthesis of CPs, including chemical routes, electrochemical deposition and photo-induced polymerization. These approaches have their own advantages and drawbacks (Figure 4).

\section{Electrochemical routes}

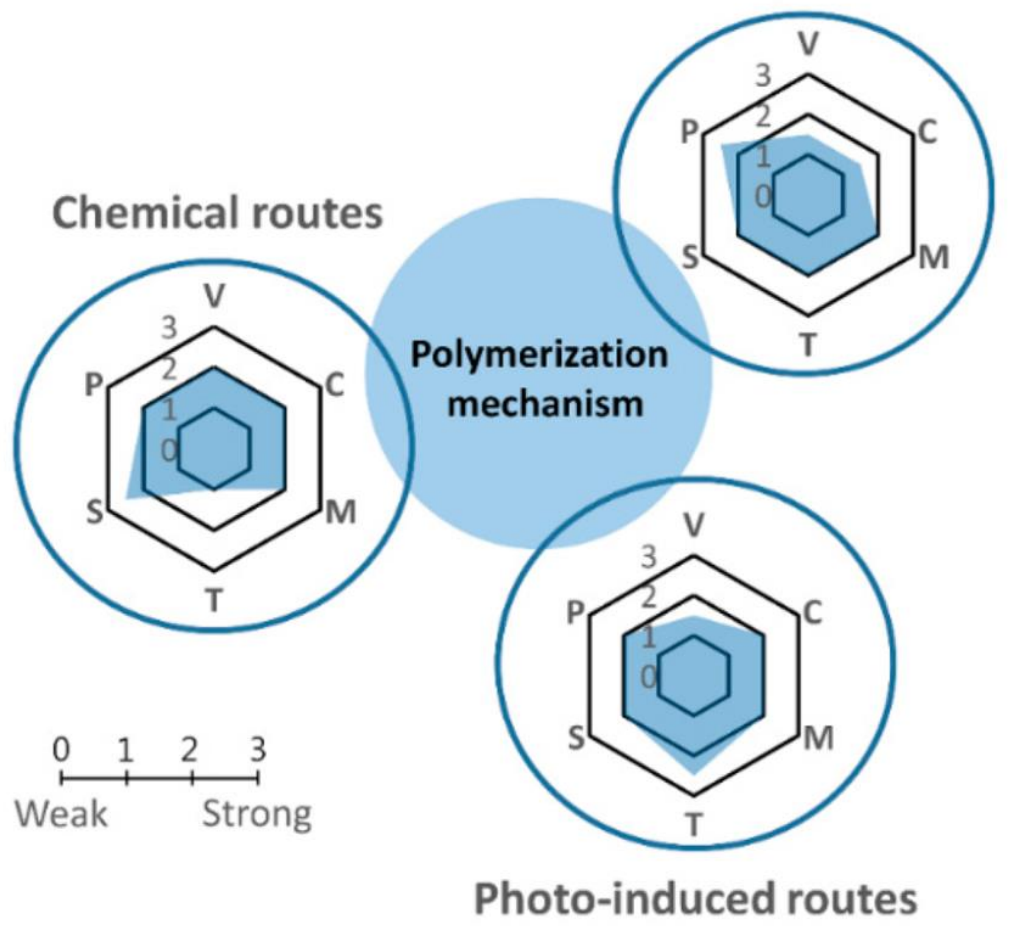

Figure 4. Schematic comparison of the main methods for CPs synthesis in terms of relative number of key variables in the synthesis process $(\mathrm{V})$, cost effectiveness $(\mathrm{C})$, morphology control $(\mathrm{M})$, reaction time (T), scalability (S), and the product purity (P). Adapted from [28].

Non-homogeneous structure of thin $\mathrm{CP}$ layer on the Gr/CP interface negatively affects the overall conductivity of the composite material $[12,29]$. According to the multi-core model proposed by Tanaka et el. [30], the structure of Gr-CP/electrolyte interfaces consists of three layers: the bounded layer, where CP and graphene sheets are interconnected by covalent, ionic 
[Type the document title]

October 8, 2017

or electrostatic forces; the interfacial region, which interacts with the surface of the nanomaterials, and the external loosely coupled layer with enhanced mobility and conformational changes of the CP chain [24]. These structural features of multiple interfaces are illustrated in Figure 5. Variation of the interfacial layer structures during charge/discharge polarization must occur in many cases and plays a critical role in charge-storing performance as well as stability of $\mathrm{Gr} / \mathrm{CP}$ composites and devices, since both double-layer capacitance and pseudocapacitance originate in the near-surface phenomena. As reported by Istam et al. [29], for example, the structure of Gr sheets uniformly covered with CP followed by subsequent stacking in a parallel manner can be denoted as series of parallel resistance-capacitor components. However, such a structure leads to additional dielectric loss [29] and should be replaced with more effective architecture for achieving better performance.



Figure 5. Schematic illustration of the interfaces between the graphene-polymer composite and electrolyte reproduced from [24]. 
[Type the document title]

October 8, 2017

High specific capacitance values of $520 \mathrm{~F} \mathrm{~g} \mathrm{~g}^{-1}$ and $253 \mathrm{~F} \mathrm{~g}^{-1}$ were achieved, when PAni nanowires or PPy nanotubes, respectively, were incorporated in rGO [31] or GO sheets [32] acting as spacers. These values can be duplicated using additional coverage of Gr with CP nanoparticles [33]. Incorporation of hollow PPy nanoparticles leads to a specific capacitance of about $500 \mathrm{~F} \mathrm{~g}^{-1}$ with excellent stability at $5 \mathrm{~A} \mathrm{~g}^{-1}$ after 10000 charge-discharge cycles [34]. Freestanding sandwich-structured $\mathrm{rGO} / \mathrm{PAni} / \mathrm{rGO}$ nanocomposite paper reported by Xiao et al. has reached a specific capacitance of $580 \mathrm{~F} \mathrm{~g}^{-1}$ with a good perspective for scalable fabrication, outperforming many analogous composites [35].

Fabrication of multilayered composites consisting of the Gr/CP bilayers is another strategy to create regular structures with attractive properties. For example, multilayer films of PAni and GO were deposited on indium tin oxide (ITO) surface exhibiting specific capacitance of $429 \mathrm{~F}$ $\mathrm{g}^{-1}$ [36]. However, it should be noted that increasing the number of layers could lead to the decrease of specific capacitance [24]. Figure 6 highlights some intriguing fabrication methods and structures of CP-Gr composites obtained by different architectures. 
[Type the document title]

October 8, 2017

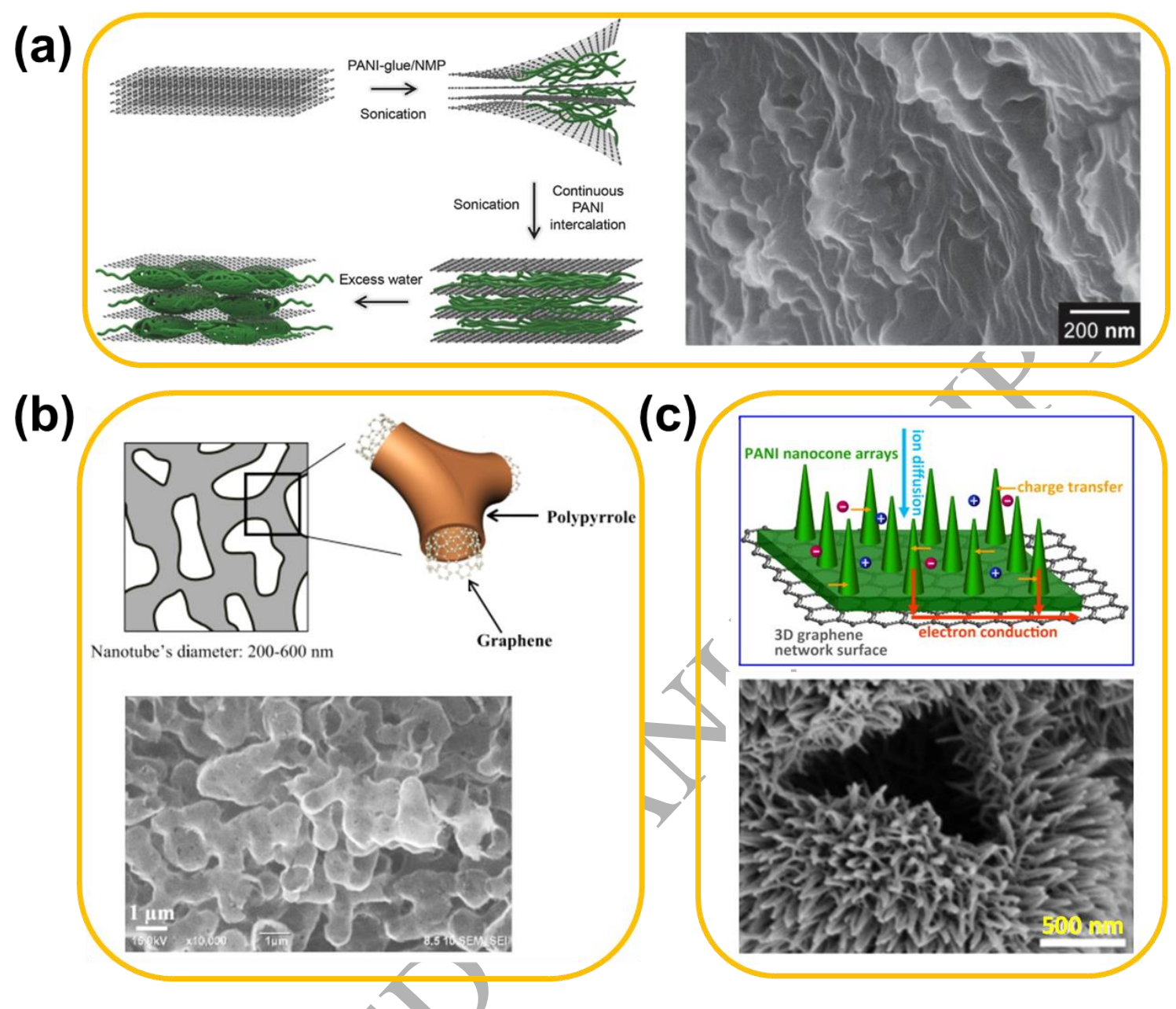

Figure 6. Schematic representation and SEM images of different $\mathrm{Gr} / \mathrm{CP}$ nanocomposite architectures synthesized by: (a) incorporation of CP between rGO sheets [37], (b) synthesis of uniform CP layer on the 3D Gr surface [38] and (c) fabrication of 3D CP nanofeatures on Gr surface $[39,40]$.

Highly developed graphene surface is a common platform to fabricate $\mathrm{Gr} / \mathrm{CP}$ composites. However, randomized 3D geometry may produce a tortuous path for diffusion of ions in the nanocomposite structure and consequently cause irreversible capacitance loss. This means that the pore size should match optimal relationship between the maximum surface area and the sizes of ions in the electrolyte [41]. Hierarchically ordered Gr/CP structures with continuous conductive skeleton display significantly higher specific capacitance compared to the intermittent designs. Wrapping of PAni nanofibers inside the rGO skeleton allows achievement of tight contact between $\mathrm{rGO}$ and $\mathrm{CP}$, resulting in a high capacitance of $921 \mathrm{~F} \mathrm{~g}^{-1}$ [42]. Bicontinuous nanotubular graphene-PPy hybrids synthesized using a nanoporous $\mathrm{Ni}$ template to create a stable 3D morphology with subsequent etching of the metal part display 
[Type the document title]

October 8, 2017

capacitance up to $509 \mathrm{~F} \mathrm{~g}^{-1}$ with excellent cycling stability [43], whereas bicontinuous $\mathrm{Gr} / \mathrm{PAni}$ composite exhibits outstanding areal capacitance of over $1.7 \mathrm{~F} \mathrm{~cm}^{-2}$ [44]. Utilization of polymer particles as an alternative to the metal removable template looks promising taking into account charge-storing ability, but the operation stability of this type of nanocomposite still needs to be improved [44-46]. Employment of $\mathrm{MnO}_{2}$ nanowires simultaneously acting as a template and oxidant for the rGO/PAni synthesis results in high capacitance of $956 \mathrm{~F} \mathrm{~g}^{-1}$ [47]. It should be noted that other materials with highly developed surface area, such as kitchen sponge [48], can also be used as a template for Gr/PAni synthesis with similar values of capacitance. Utilization of the 3D sponge-like ionic liquid-graphene assembly as a matrix for $\mathrm{CP}$ electropolymerization demonstrates capacitance up to $662 \mathrm{~F} \mathrm{~g}^{-1}$ with excellent cycling stability of about $93 \%$ at $10 \mathrm{~A} \mathrm{~g}^{-1}$ after 5000 consecutive cycles [49]. Fabrication of PAnimodified 3D graphene frameworks with rapid mixing of the oxidant and monomer solutions allows avoiding the CP film overgrowth and achieving extraordinarily high specific capacitance and energy density, $\sim 1000 \mathrm{~F} \mathrm{~g}^{-1}$ and $\sim 120 \mathrm{~W} \mathrm{~h} \mathrm{~kg}^{-1}$, respectively [50], which is the highest energy density achieved so far for all Gr-based nanocomposites for pseudo-SCs [51]. These findings are in good relationship with results of the recent study of Gr/PAni layered composites with different morphology, where pore connectivity and accessibility to the electrolyte was shown as the dominant factor determining fast ion transport and kinetics of the pseudo faradaic redox process [52].

Employment of Gr surface as a conductive support to create nanostructured $\mathrm{CP}$ architectures is also a perspective strategy to fabricate freestanding flexible materials with outstanding chargestoring ability. rGO/PAni paper exhibits enhanced ion diffusion due to the shortened pathways of ion migration compared to the pristine PAni and high capacitance of $763 \mathrm{~F} \mathrm{~g}^{-1}$ [53] and 939 $\mathrm{F} \mathrm{g}^{-1}$ for controllably grown PAni nanowires [40] or $751.3 \mathrm{~F} \mathrm{~g}^{-1}$ for PAni nanocones [39]. Similar performance was achieved when low temperature oxidation polymerization method was used on the 4-aminophenyl pre-crafted rGO [54] and further improved by utilization of the sulfonated triazine to initiate the growth of PAni nanorods [55]. Another nanocomposite of Gr nanomesh coated with PAni nanorods exhibits extraordinarily high-rate capability with 98\% of capacitance retention when current density was increased from $1 \mathrm{~A} \mathrm{~g}^{-1}$ to $50 \mathrm{~A} \mathrm{~g}^{-1}$ [56]. It should be mentioned that $\mathrm{Gr}$ itself does not contribute significantly to the overall capacitance of this type of materials; instead, the Gr matrix mainly provides attractive mechanical and 
[Type the document title]

October 8, 2017

electrical properties to fabricate the freestanding SC electrodes. Therefore, similar capacitance values can be achieved using alumina oxide [57] or stainless steel [58] substrates.

Recent achievements in the specific capacitance of $\mathrm{Gr} / \mathrm{CP}$ nanocomposites described above indicate a limiting value of $c a .1000 \mathrm{~F} \mathrm{~g}^{-1}$, which is close to the best results obtained at those SCs electrodes based on metal oxides and hydroxides [59, 60], confirming the competent capability of $\mathrm{Gr} / \mathrm{CP}$ nanocomposites among mostly targeted materials for electrochemical energy-storing purposes.

\subsection{Applications of Gr/CP nanocomposites in flexible supercapacitors}

Materials based on Gr/CP nanocomposites are attractive candidates for high-performance thin and flexible microsystems for energy storage, which can be used as a lightweight power source for portable electronics, electronic papers, bendable displays, etc. [20, 61-63].

Utilization of PAni nanofibers wrapped with rGO sheets results in a volumetric capacitance of $25.6 \mathrm{~F} \mathrm{~cm}^{-3}$ at $0.12 \mathrm{~A} \mathrm{~cm}^{-3}$ with excellent cycling stability without structural failure and loss of performance in their bended state [42]. In another example, flexible all-solid-state SCs based on the GO/PAni composites were construeted [64]. Resulting wave-shaped bendable and stretchable SCs exhibited the maximum specific capacitance up to $1095 \mathrm{~F} \mathrm{~g}^{-1}$ at $1 \mathrm{~A} \mathrm{~g} \mathrm{~g}^{-1}$ with an energy density of $24.3 \mathrm{~W} \mathrm{~h} \mathrm{~kg}^{-1}$ and a maximum power density of $28.1 \mathrm{~kW} \mathrm{~kg}^{-1}$ (Figure 7). The SCs have excellent retention of properties after mechanical treatment (Figure $7 c)$.

Assembled flexible and all-sølid-state supercapacitor employed rGO paper with incorporated PANI-PSS nanoparticles, has a volumetric specific capacitance of $217 \mathrm{~F} \mathrm{~cm}^{-3}$ at $0.37 \mathrm{~A} \mathrm{~cm}^{-3}$ [65], which is significantly larger than the values obtained for CNTs/CP-based designs [66, 67] and close to the value obtained for the symmetrical SC based on the 3D foam of rGO/PAni nanowires [68]. Bendable micro-SC comprised of the electrodes fabricated by incorporation of PAni nanofibers between $\mathrm{rGO}$ sheets also offered remarkable volumetric capacitance of $436 \mathrm{~F}$ $\mathrm{cm}^{-3}$ at $10 \mathrm{mV} \mathrm{s}^{-1}$ [69]. 
[Type the document title]

October 8, 2017

(a)

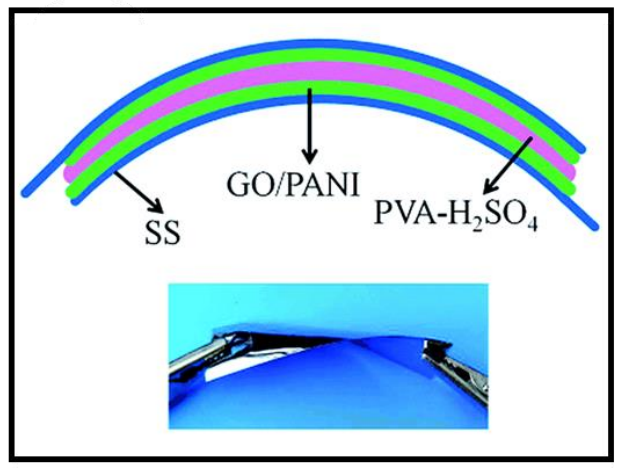

(c)

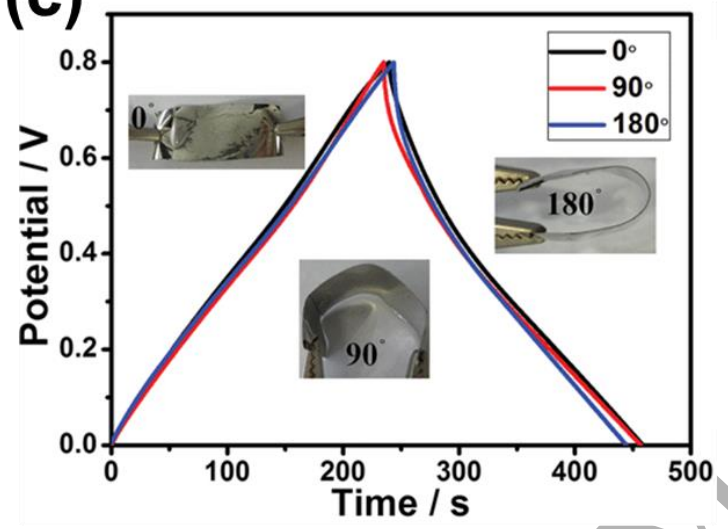

(b)

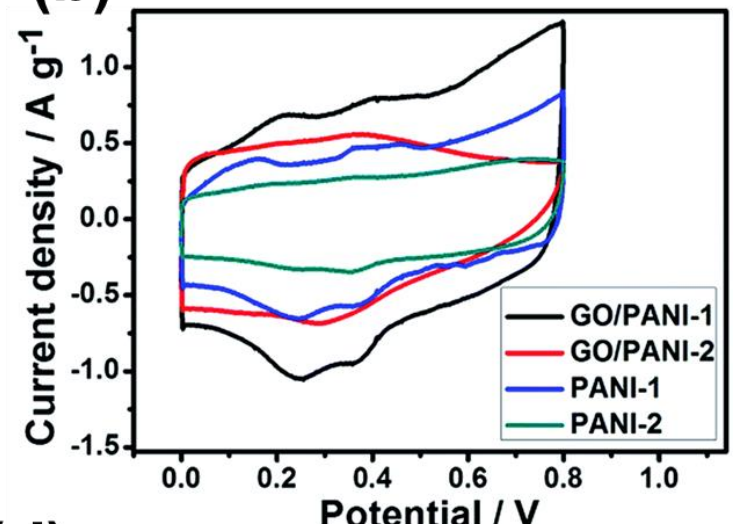

(d)

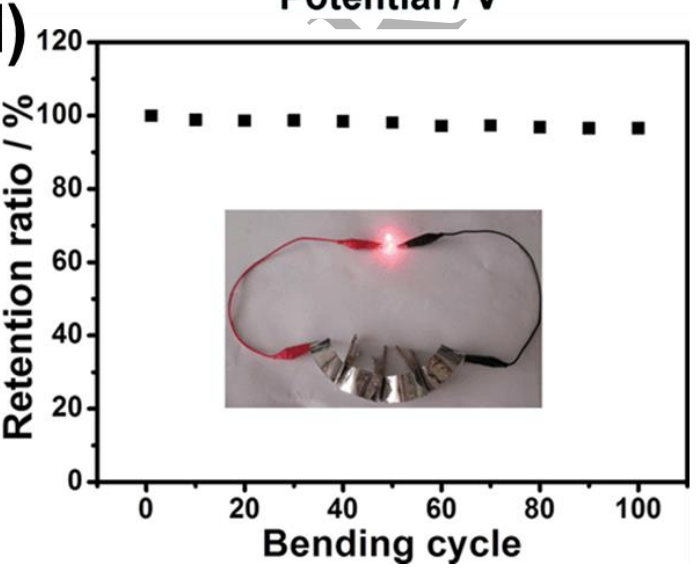

Figure 7. Example of graphene-conducting polymer composite based flexible and bendable SCs: (a) schematic representation of the structure and optical image, (b) comparison of cyclic voltammograms (CVs) of various materials obtained at $5 \mathrm{mV} \mathrm{s}^{-1}$, (c) comparison of Galvanostatic charge-discharge curves $\left(1 \mathrm{~A} \mathrm{~g} \mathrm{~g}^{-1}\right)$ of GO/PANI based flexible supercapacitors obtained at various bending angles, and (d) evaluations on the bending cycle stability of GO/PANI based SCs. Insets in (c) are photographs of bended SCs with different angles, and inset in (d) is an LED illuminated by the device units connected in series. Adapted from Ref. [64].

Micro-SCs based on sandwich-like layered mesoporous Gr/CP nanosheets with uniform and densely packed structure can deliver a volumetric capacitance of $736 \mathrm{~F} \mathrm{~cm}^{-3}$ and $436 \mathrm{~F} \mathrm{~cm}^{-3}$ at $10 \mathrm{mV} \mathrm{s}^{-1}$, respectively using water and poly(vinyl alcohol) in electrolyte [70]. This is the highest specific capacitance obtained at 2D thin-film micro-SCs, regardless of electrode materials [71].

In addition to fabrication of SCs, attractive pseudocapacitive properties of $\mathrm{Gr} / \mathrm{CP}$ materials can also be used in other systems or devices, for example the devices for capacitive water deionization with an enhanced salt removal performance [72].

\section{Perspective and challenges}


[Type the document title]

October 8, 2017

Perspectives of the further development of $\mathrm{Gr} / \mathrm{CP}$ nanomaterials for the capacitive energy storage purposes can be related to two main directions. The first one is concerned with the improvement of intrinsic properties of $\mathrm{Gr} / \mathrm{CP}$ nanomaterials, e.g. utilization of new derivatives, copolymers or even more complex conjugated structures, e.g. proteins [73], introduction of additional components to the $\mathrm{Gr} / \mathrm{CP}$ nanocomposites, development of novel architectures, methods of synthesis etc. Recent advances in this field rest on the use of additional carbon or metal nanomaterials to facilitate electron transfer process and improve capacitive performance $[74,75]$.

The second direction is related to a variety of ways using complete Gr/CP-based electrodes in SCs. Combination of nanocomposite-based electrodes into asymmetric SCs, which display superior properties compared to the symmetric design [76], or to more complex battery-SC hybrids [77, 78], leads to significantly higher energy density and voltage compared to the conventional approach.

The appealing properties of $\mathrm{Gr} / \mathrm{CP}$ nanocomposites can also be utilized in currently expanding field of dual-feature self-charging SC/power cell hybrids for mechanical- [79, 80], chemical[81] or solar-to-electric energy conversion [82] coupled with electrochemical energy storage within a singular contrivance. Nanostructured Gr/CP surfaces, being a promising matrix for the entrapment of various bioelements (living cells [83], protein complexes [84], redox enzymes [85], etc.), offer further opportunities for the development of supercapacitive biofuel cells [86, 87] and photo-bioelectrochemical cells [88] (in other words, self-charging biosupercapacitors (BioSCs) and photo-BioSCs) with improved performance and prospects for miniaturization of devices.

Alternative application of $\mathrm{Gr} / \mathrm{CP}$ materials is not limited to particular capacitive storage of electrie charges but expanding to the broad field of energy storage. For instance, $\mathrm{Gr} / \mathrm{CP}$ nanocomposites were successfully utilized in various types of organic batteries to demonstrate promising features [89].

In spite of the numerous advantages of many $\mathrm{Gr} / \mathrm{CP}$ composites, complicated procedures of synthesis and limited ability for mass production remain a bottleneck for their broad applications. More recently, some cost-effective and template-free methods for the scale-up or mass fabrication of 3D Gr frameworks under mild conditions have been proposed [90, 91]. A number of composites, fabricated by further modification of the structures with CPs, have 
[Type the document title]

October 8, 2017

shown promising performances. These latest advances may open new ways to use $\mathrm{Gr} / \mathrm{CP}$ nanostructured composite materials for the development of inexpensive and scalable capacitive charge-storing devices.

\section{Acknowledgements}

This work was supported by the Danish Council for Independent Research-Technology and Product Sciences (DFF-FTP, Project No. 12-127447, to Q.C.). F.S. acknowledges the PhD scholarships offered by China Scholarship Council (CSC 201506170059). D.P. is grateful for the Ørsted-Cofunded postdoc fellowship.

\section{References and Recommended reading}

These papers of particular interest, published within the period of this review and recommended for further reading, have been highlighted as:

- Paper of special interest

•- Paper of outstanding interest

1. Burke A: Ultracapacitor; Why, and Where is the technology. J. Power Sources 2000, 91:37-50.

2. Cao X, Yin Z, Zhang H: Three-dimensional graphene materials: preparation, structures and application in supercapacitors. Energy Environ. Sci. 2014, 7:18501865 .

3. González A, Goikolea E, Barrena JA, Mysyk R: Review on supercapacitors: Technologies and materials. Renew. Sustain. Energy Rev. 2016, 58:1189-1206.

4. Madou MJ: Fundamentals of microfabrication and nanotechnology. CRC Press; 2012.

5. Kotz R; Carlen M.: Principles and applications of electrochemical capacitors. Electrochim. Acta 2000, 45: 2483-98.

6. Simon P, Gogotsi Y: Materials for electrochemical capacitors. Nat. Mater. 2008, 7:845-854.

7. Pandolfo AG, Hollenkamp AF: Carbon properties and their role in supercapacitors. J. Power Sources 2006, 157:11-27.

8. Pieta P, Obraztsov I, D'Souza F, Kutner W: Composites of conducting polymers and various carbon nanostructures for electrochemical supercapacitors. ECS J. Solid State Sci. Technol. 2013, 2:M3120-M3134.

9. Scherer MRJ: Double-gyroid-structured functional materials. Springer International Publishing; 2013.

10. Meng Q, Cai K, Chen Y, Chen L: Research progress on conducting polymer based Page 18 of 24 
[Type the document title]

October 8, 2017

supercapacitor electrode materials. Nano Energy 2017, 36:268-285.

11. MacDiarmid AG, Mammone RJ, Kaner RB, Porter SJ, Pethig R, Heeger AJ, Rosseinsky DR: The concept of 'doping' of conducting polymers: the role of reduction potentials. Philos. Trans. R. Soc. A Math. Phys. Eng. Sci. 1985, 314:3-15.

12. Hashemi R, Weng GJ: A theoretical treatment of graphene nanocomposites with percolation threshold, tunneling-assisted conductivity and microcapacitor effect in AC and DC electrical settings. Carbon N. Y. 2016, 96:474-490.

13. Xia J, Chen F, Li J, Tao N: Measurement of the quantum capacitance of graphene. Nat. Nanotechnol. 2009, 4:505-509.

14. Dey RS, Hjuler HA, Chi Q, Tao N, Ji H, Murali S, Wu Y, Perales S, Clevenger B, Ruoff RS, et al.: Approaching the theoretical capacitance of graphene through copper foam integrated three-dimensional graphene networks. J. Mater. Chem. A 2015, 3:6324-6329.

15. Peng Z, Ye R, Mann JA, Zakhidov D, Li Y, Smalley PR, Lin J, Tour JM: Flexible boron-doped laser-induced graphene microsupercapacitors. ACS Nano 2015, 9:5868-5875.

16. Ji L, Meduri P, Agubra V, Xiao X, Alcoutlabi M: Graphene-based nanocomposites for energy storage. Adv. Energy Mater. 2016, 6: 1502159.

17. Mahmood N, Zhang C, Yin H, Hou Y: Graphene-based nanocomposites for energy storage and conversion in lithium batteries, supercapacitors and fuel cells. $J$. Mater. Chem. A 2014, 2:15-32.

18. Worfolk BJ, Andrews SC, Park S, Reinspach J, Liu N, Toney MF, Mannsfeld SCB, Bao Z: Ultrahigh electrical conductivity in solution-sheared polymeric transparent films. . Proc. Natl. Acad. Sci. U. S. A. 2015, 112:14138-43.

19. Li H, Wang J, Chu Q, Wang Z, Zhang F, Wang S: Theoretical and experimental specific capacitance of polyaniline in sulfuric acid. J. Power Sources 2009, 190:578-586.

20. Kim BC, Hong JY, Wallace GG, Park HS: Recent progress in flexible electrochemical capacitors: electrode materials, device configuration, and functions. Adv. Energy Mater. 2015, 5.

21. Zhao J, Wu J, Li B, Du W, Huang Q, Zheng M, Xue H, Pang H: Facile synthesis of polypyrrole nanowires for high-performance supercapacitor electrode materials. Prog. Nat. Sci. Mater. Int. 2016, 26:237-242.

22. Kim M, Lee C, Jang J: Fabrication of highly flexible, scalable, and highperformance supercapacitors using polyaniline/reduced graphene oxide film with enhanced electrical conductivity and crystallinity. Adv. Funct. Mater. 2014, 24:2489-2499.

23. Zhang J, Zhao XS: On the configuration of supercapacitors for maximizing electrochemical performance. ChemSusChem 2012, 5:818-841.

- 24. Gupta S, Price C: Investigating graphene/conducting polymer hybrid layered composites as pseudocapacitors: Interplay of heterogeneous electron transfer, electric double layers and mechanical stability. Compos. Part B Eng. 2016, 105:46- 
[Type the document title]

October 8, 2017

59.

Important to support the hypothesis that the surface morphology is influental with electrical conductivity of nanocomposites.

25. Punetha VD, Rana S, Yoo HJ, Chaurasia A, McLeskey JT, Ramasamy MS, Sahoo NG, Cho JW: Functionalization of carbon nanomaterials for advanced polymer nanocomposites: A comparison study between CNT and graphene. Prog. Polym. Sci. 2017, 67:1-47.

26. Wang M, Duan X, Xu Y, Duan X: Functional three-dimensional graphene/polymer composites. ACS Nano 2016, 10:7231-7247.

-27. Wang M, Xu YX: Design and construction of three-dimensional graphene/conducting polymer for supercapacitors. Chinese Chem. Lett. 2016, 27:1437-1444.

Important as an overview of recent advances in developing effective strategies to synthesize 3D Gr/CP composites for energy storage applications.

28. Nguyen DN, Yoon $\mathrm{H}$ : Recent advances in nanostructured conducting polymers: From synthesis to practical applications. Polymers (Basel). 2016, 8.

29. Islam R, Papathanassiou AN, Chan Yu King R, Brun J-F, Roussel F: Evidence of interfacial charge trapping mechanism in polyaniline/reduced graphene oxide nanocomposites. Appl. Phys. Lett. 2015, 107:53102.

30. Tanaka T, Kozako M, Fuse N, Ohki Y: Proposal of a multi-core model for polymer nanocomposite dielectrics. IEEE Trans. Dielectr. Electr. Insul. 2005, 12:669-681.

31. Yang F, Xu M, Bao S-J, Wei H, Chai H: Self-assembled hierarchical graphene/polyaniline hybrid aerogels for electrochemical capacitive energy storage. Electrochim. Acta 2014, 137:381-387.

32. Ye S, Feng J: Self-assembled three-dimensional hierarchical graphene/polypyrrole nanotube hybrid aerogel and its application for supercapacitors. ACS Appl. Mater. Interfaces 2014, 6:9671-9679.

33. Yan J, Wei T, Shao B, Fan Z, Qian W, Zhang M, Wei F: Preparation of a graphene nanosheet/polyaniline composite with high specific capacitance. Carbon N. Y. 2010, 48:487-493.

34. Zhang J, Yu Y, Liu L, Wu Y, Ren J, Tang FQ, Wu W: Graphene-hollow PPy sphere 3D-nanoarchitecture with enhanced electrochemical performance. Nanoscale 2013, 5:3052.

- 35. Xiao F, Yang S, Zhang Z, Liu H, Xiao J, Wan L, Luo J, Wang S, Liu Y: Scalable synthesis of freestanding sandwich-structured graphene/polyaniline/graphene nanocomposite paper for flexible all-solid-state supercapacitor. Sci. Rep. 2015, 5:9359.

Important study demonstrating scalable method to fabricate a unique sandwich-structured graphene/polyaniline/graphene paper.

36. Mitchell E, Candler J, De Souza F, Gupta RK, Gupta BK, Dong LF: High performance supercapacitor based on multilayer of polyaniline and graphene 
[Type the document title]

October 8, 2017

oxide. Synth. Met. 2015, 199:214-218.

37. Choi H, Ahn K-J, Lee Y, Noh S, Yoon H: Free-standing, multilayered graphene/polyaniline-glue/graphene nanostructures for flexible, solid-state electrochemical capacitor application. Adv. Mater. Interfaces 2015, 2:1500117.

38. Kashani H, Chen L, Ito Y, Han J, Hirata A, Chen M: Bicontinuous nanotubular graphene-polypyrrole hybrid for high performance flexible supercapacitors. Nano Energy 2016, 19:391-400.

39. Yu M, Ma Y, Liu J, Li S: Polyaniline nanocone arrays synthesized on threedimensional graphene network by electrodeposition for supercapacitor electrodes. Carbon N. Y. 2015, 87:98-105.

40. Yu P, Zhao X, Li Y, Zhang Q: Controllable growth of polyaniline nanowire arrays on hierarchical macro/mesoporous graphene foams for high-performance flexible supercapacitors. Appl. Surf. Sci. 2017, 393:37-45.

- 41. Yang J, Liu Y, Liu S, Li L, Zhang C, Liu T: Conducting polymer composites: material synthesis and applications in electrochemical capacitive energy storage. Mater. Chem. Front. 2017, 1:251-268.

Important as a recent comprehensive overview of conducting polymer-based nanocomposite materials.

42. Hu N, Zhang L, Yang C, Zhao J, Yang Z, Wei H, Liao H, Feng Z, Fisher A, Zhang Y, et al.: Three-dimensional skeleton networks of graphene wrapped polyaniline nanofibers: an excellent structure for high-performance flexible solid-state supercapacitors. Sci. Rep. 2016, 6:19777.

43. Zhang J, Wang J, Yang J, Wang Y, Chan-Park MB: Three-dimensional macroporous graphene foam filled with mesoporous polyaniline network for high areal capacitance. ACS Sustain. Chem. Eng. 2014, 2:2291-2296.

44. Fan W, Zhang C, Tjiu WW, Pramoda KP, He C, Liu T: Graphene-wrapped polyaniline hollow spheres as novel hybrid electrode materials for supercapacitor applications. ACS Appl. Mater. Interfaces 2013, 5:3382-3391.

45. Trung NB, Tam T Van, Kim HR, Hur SH, Kim EJ, Choi WM: Three-dimensional hollow balls of graphene-polyaniline hybrids for supercapacitor applications. Chem. Eng. J. 2014, 255:89-96.

46. Luo J, Ma Q, Gu H, Zheng Y, Liu X: Three-dimensional graphene-polyaniline hybrid hollow spheres by layer-by-layer assembly for application in supercapacitor. Electrochim. Acta 2015, 173:184-192.

-• 47. Yang C, Zhang L, Hu N, Yang Z, Su Y, Xu S, Li M, Yao L, Hong M, Zhang Y:

Rational design of sandwiched polyaniline nanotube/layered graphene/polyaniline nanotube papers for high-volumetric supercapacitors. Chem. Eng. J. 2017, 309:89-97.

Unique as a study demonstrating a rational design of graphene-polyaniline films with outstanding electrochemical performance synthesized by $\mathrm{MnO}_{2}$-templated polymerization.

48. Moussa M, El-Kady MF, Wang H, Michimore A, Zhou Q, Xu J, Majeswki P, Ma J: 
[Type the document title]

October 8, 2017

High-performance supercapacitors using graphene/polyaniline composites deposited on kitchen sponge. Nanotechnology 2015, 26:75702.

49. Halab Shaeli Iessa K, Zhang Y, Zhang G, Xiao F, Wang S: Conductive porous sponge-like ionic liquid-graphene assembly decorated with nanosized polyaniline as active electrode material for supercapacitor. J. Power Sources 2016, 30: 92-97.

50. Kulkarni SB, Patil UM, Shackery I, Sohn JS, Lee S, Park B, Jun S, Guo J, Lian K: High-performance supercapacitor electrode based on a polyaniline nanofibers/3D graphene framework as an efficient charge transporter. J. Mater. Chem. A 2014, 2:4989.

51. Xiong $\mathrm{P}$, Zhu J, Zhang L, Wang X: Recent advances in graphene-based hybrid nanostructures for electrochemical energy storage. Nanoscale Horiz. 2016, 1:340374.

•• 52. Wang Y, Yang X, Pandolfo AG, Ding J, Li D: High-rate and high-volumetric capacitance of compact graphene-polyaniline hydrogel electrodes. Adv. Energy Mater. 2016, 6:1600185.

This paper is unique demonstrating the excellent charge-storing ability of Gr/PAni hydrogel electrodes achieved through well-maintained pore connectivity.

53. Cong HP, Ren XC, Wang P, Yu SH: Flexible graphene-polyaniline composite paper for high-performance supercapacitor. Energy Environ. Sci. 2013, 6:1185.

54. Liu X, Shang P, Zhang Y, Wang X, Fan Z, Wang B, Zheng Y: Three-dimensional and stable polyaniline-grafted graphene hybrid materials for supercapacitor electrodes. J. Mater. Chem. A 2014, 2:15273.

55. Liu Y, Ma Y, Guang S, Xu H, Su X, Tan J, Wu ZS, Gentle I, Lu GQ, Cheng HM: Facile fabrication of three-dimensional highly ordered structural polyanilinegraphene bulk hybrid materials for high performance supercapacitor electrodes. J. Mater. Chem. A 2014, 2:813-823.

56. Gao S, Zang P, Dang L, Xu H, Shi F, Liu Z, Lei Z: Extraordinarily high-rate capability of polyaniline nanorod arrays on graphene nanomesh. J. Power Sources 2016, 304:111-118.

57. Zhao G-Y, Li H-L: Preparation of polyaniline nanowire arrayed electrodes for electrochemical supercapacitors. Micropor. Mesopor. Mat. 2008, 110:590-594.

58. Dhawale DS, Dubal DP, Jamadade VS, Salunkhe RR, Lokhande CD: Fuzzy nanofibrous network of polyaniline electrode for supercapacitor application. Synth. Met. 2010, 160:519-522.

59. Augustyn V, Simon P, Dunn B: Pseudocapacitive oxide materials for high-rate electrochemical energy storage. Energy Environ. Sci. 2014, 7:1597.

60. Yan W, Kim JY, Xing W, Donavan KC, Ayvazian T, Penner RM: Lithographically patterned gold/manganese dioxide core/shell nanowires for high capacity, high rate, and high cyclability hybrid electrical energy storage. Chem. Mater. 2012, 24:2382-2390.

61. $\mathrm{Hu} \mathrm{H}$, Pei Z, Ye C: Recent advances in designing and fabrication of planar microsupercapacitors for on-chip energy storage. Energy Storage Mater. 2015, 1:82-102. 
[Type the document title]

October 8, 2017

62. Liu W, Song M-S, Kong B, Cui Y: Flexible and stretchable energy storage: Recent advances and future perspectives. Adv. Mater. 2017, 29:1603436.

63. Lv W, Li Z, Deng Y, Yang Q-H, Kang F: Graphene-based materials for electrochemical energy storage devices: Opportunities and challenges. Energy Storage Mater. 2016, 2:107-138.

•• 64. Li D, Li Y, Feng Y, Hu W, Feng W, Dou SX, Qiu KW, Li CM, Yu T, Cheng HM: Hierarchical graphene oxide/polyaniline nanocomposites prepared by interfacial electrochemical polymerization for flexible solid-state supercapacitors. J. Mater. Chem. A 2015, 3:2135-2143.

This work presents impressive observations on graphene oxide/polyaniline composites for flexible and bendable solid-state supercapacitors that can resist to multi-cycle bending without loss of supercapative storing capacibility.

65. Yang C, Zhang L, Hu N, Yang Z, Wei H, Xu ZJ, Wang Y, Zhang Y: Densely-packed graphene/conducting polymer nanoparticle papers for high-yolumetricperformance flexible all-solid-state supercapacitors. Appl. Surf. Sci. 2016, 379:206212.

66. de Souza VHR, Oliveira MM, Zarbin AJG: Thin and flexible all-solid supercapacitor prepared from novel single wall carbon nanotubes/polyaniline thin films obtained in liquid-liquid interfaces. J. Power Sources 2014, 260:34-42.

67. Chen Y, Du L, Yang P, Sun P, Yu X, Mai W: Significantly enhanced robustness and electrochemical performance of flexible carbon nanotube-based supercapacitors by electrodepositing polypyrrole. J. Power Sources 2015, 287:68-74.

68. Yu P, Zhao X, Huang Z, Li Y, Zhang Q, Jing X, Wang B, Zhang M, Liu L, deHeer WA, et al.: Free-standing three-dimensional graphene and polyaniline nanowire arrays hybrid foams for high-performance flexible and light weight supercapacitors. J. Mater. Chem. A 2014, 2:14413.

69. Mao L, Li M, Xue J, Wang J: Bendable graphene/conducting polymer hybrid films for freestanding electrodes with high volumetric capacitances. RSC Adv. 2016, 6:2951-2957.

- 70. Wu ZS, Parvez K, Li S, Yang S, Liu Z, Liu S, Feng X, Müllen K: Alternating stacked graphene-conducting polymer compact films with ultrahigh areal and volumetric capacitances for high-energy micro-supercapacitors. Adv. Mater. 2015, 27:4054-4061.

Important to demonstrate the unique alternating layered structure of Gr/PAni nanocomposite for microSCs with superior performance.

- 71. Kyeremateng NA, Brousse T, Pech D: Microsupercapacitors as miniaturized energy-storage components for on-chip electronics. Nat. Nanotechnol. 2017, 12:715 .

This review provides an invaluable description of the state-of-the-art of microsupercapacitors until 2016.

72. Gu X, Yang Y, Hu Y, Hu M, Huang J, Wang C, Kim DK, Cao AQ, Qu LT, Yuan Q, et al.: Facile fabrication of graphene-polypyrrole-Mn composites as high- 
[Type the document title]

October 8, 2017

performance electrodes for capacitive deionization. J. Mater. Chem. A 2015, 3:5866-5874.

•• 73. Mosa IM, Pattammattel A, Kadimisetty K, Pande P, El-Kady MF, Bishop GW, Novak M, Kaner RB, Basu AK, Kumar C V., et al.: Ultrathin graphene-protein supercapacitors for miniaturized bioelectronics. Adv. Energy Mater. 2017, 1700358.

Paper is unique by demonstrating the possiblilty to utilize a layered nanocomposite of graphene with human proteins for charge storing purposes.

74. Kalambate PK, Dar RA, Karna SP, Srivastava AK: High performance supercapacitor based on graphene-silver nanoparticles-polypyrrole nanocomposite coated on glassy carbon electrode. J. Power Sources 2015, 276:262270.

75. Pan C, Gu H, Dong L: Synthesis and electrochemical performance of polyaniline $@ \mathrm{MnO}_{2} /$ graphene ternary composites for electrochemical supercapacitors. $J$. Power Sources 2016, 303:175-181.

76. Kurra N, Wang R, Alshareef HN, Casas MT, Ferreirae CA, Alemán C, Tong Y, Li Y, Li Y, Ajayan PM: All conducting polymer electrodes for asymmetric solid-state supercapacitors. J. Mater. Chem. A 2015, 3:7368-7374.

77. Choi HS, Park CR: Theoretical guidelines to designing high performance energy storage device based on hybridization of lithium-ion battery and supercapacitor. J. Power Sources 2014, 259:1-14.

78. Dubal DP, Ayyad O, Ruiz V, Gómez-Romero P, Zhang Y, Tang H, Gu C, Wang X, Tu $\mathrm{J}$ : Hybrid energy storage: the merging of battery and supercapacitor chemistries. Chem. Soc. Rev. 2015, 44:1777-1790.

79. Xue XY, Deng P, He B, Nie YX, Xing LL, Zhang Y, Wang ZL: Flexible selfcharging power cell for one-step energy conversion and storage. Adv. Energy Mater. 2014, 4:1301329.

80. Ramadoss A, Saravanakumar B, Lee SW, Kim YS, Kim SJ, Wang ZL: Piezoelectricdriven self-charging supercapacitor power cell. ACS Nano 2015, 9:4337-4345.

81. Pankratov D, Falkman P, Blum Z, Shleev S: A hybrid electric power device for simultaneous generation and storage of electric energy. Energy Environ. Sci. 2014, 7:989-993.

82. Sun H, You X, Deng J, Chen XL, Yang ZB, Chen PN, Fang X, Peng HS: A twisted wire-shaped dual-function energy device for photoelectric conversion and electrochemical storage. Angew. Chem., Int. Ed. 2014, 53:6664-6668.

83. Liu X, Wu W, Gu Z. Poly (3,4-ethylenedioxythiophene) promotes direct electron transfer at the interface between Shewanella loihica and the anode in a microbial fuel cell. J. Power Sources 2015, 277:110-115.

84. Li GL, Feng XY, Fei JB, Cai P, Li JA, Huang JG, Li JB: Interfacial assembly of photosystem II with conducting polymer films toward enhanced photobioelectrochemical cells. Adv. Mater. Interfaces 2017, 4:1600619.

85. Parunova YM, Bushnev SO, Gonzalez-Arribas E, Falkman P, Lipkin AV, Popov VO, Shleev SV, Pankratov DV: Potentially implantable biocathode with the function of 
[Type the document title]

October 8, 2017

charge accumulation based on nanocomposite of polyaniline/carbon nanotubes. Russ. J. Electrochem. 2016, 52:1166-1171.

86. Agnes C, Holzinger M, Le Goff A, Reuillard B, Elouarzaki K, Tingry S, Cosnier S: Supercapacitor/biofuel cell hybrids based on wired enzymes on carbon nanotube matrices: autonomous reloading after high power pulses in neutral buffered glucose solutions. Energy Environ. Sci. 2014, 7:1884-1888.

87. Xiao X, Ó Conghaile P, Leech D, Ludwig R, Magner E: An oxygen-independent and membrane-less glucose biobattery/supercapacitor hybrid device. Biosens. Bioelectron. 2017, 98:421-427.

88. Pankratova G, Pankratov D, Hasan K, Åkerlund H-E, Albertsson P-Å, Leech D, Shleev S, Gorton L: Supercapacitive photo-bioanodes and biosolar cells: A novel approach for solar energy harnessing. Adv. Energy Mat. 2017, 7:1602285.

89. Muench S, Wild A, Friebe C, Häupler B, Janoschka T, Schubert US: Polymer-based organic batteries. Chem. Rev. 2016, 116:9438-9484.

90. Yu M, Huang Y, Li C, Zeng Y, Wang W, Li Y, Fang P, Lu X, Tong Y: Building three-dimensional graphene frameworks for energy storage and catalysis. $A d v$. Funct. Mater. 2015, 25:324-330.

91. Woltornist SJ, Carrillo J-MY, Xu TO, Dobrynin A V., Adamson DH:

Polymer/pristine graphene based composites: From emulsions to strong, electrically conducting foams. Macromolecules 2015, 48:687-693. 
[Type the document title]

\section{TOC graphic abstract}

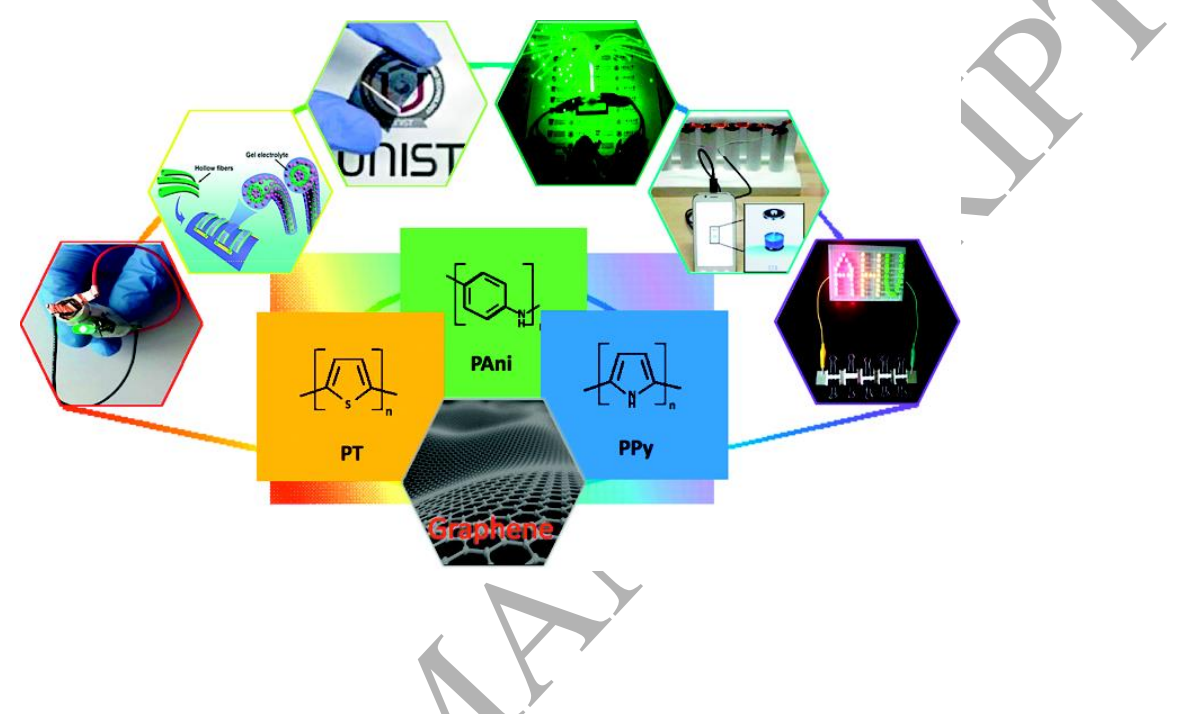

Synopsis: This minreview presents recent advances and our comments on the research subject of graphene-conducting polymer nanocompøsite based supercapacitors. We emphasize material design, controlled assembly and nanoscale engineering of relevant composites, and their applications in flexible and bendable supercapacitors. 